\title{
ALGEBRAIC HYPERSURFACES
}

\author{
JÁNOS KOLLÁR
}

\begin{abstract}
We give an introduction to the study of algebraic hypersurfaces, focusing on the problem of when two hypersurfaces are isomorphic or close to being isomorphic. Working with hypersurfaces and emphasizing examples makes it possible to discuss these questions without any previous knowledge of algebraic geometry. At the end we formulate the main recent results and state the most important open questions.
\end{abstract}

\section{Contents}

1. Stereographic projection

2. Projective hypersurfaces

3. Rational and birational maps

4. The main questions

5. Rationality of cubic hypersurfaces

6. Isomorphism of hypersurfaces

7. Nonrationality of large degree hypersurfaces

8. Nonrationality of low degree hypersurfaces

9. Rigidity of low degree hypersurfaces

10. Connections with the classification of varieties

11. Open problems about hypersurfaces

Acknowledgments

References

Algebraic geometry started as the study of plane curves $C \subset \mathbb{R}^{2}$ defined by a polynomial equation and later extended to surfaces and higher dimensional sets defined by systems of polynomial equations. Besides using $\mathbb{R}^{n}$, it is frequently more advantageous to work with $\mathbb{C}^{n}$ or with the corresponding projective spaces $\mathbb{R} \mathbb{P}^{n}$ and $\mathbb{C P}^{n}$.

Later it was realized that the theory also works if we replace $\mathbb{R}$ or $\mathbb{C}$ by other fields, for example the field of rational numbers $\mathbb{Q}$ or even finite fields $\mathbb{F}_{q}$. When we try to emphasize that the choice of the field is pretty arbitrary, we use $\mathbb{A}^{n}$ to denote affine $n$-space and $\mathbb{P}^{n}$ to denote projective $n$-space.

Received by the editors May 29, 2018.

2010 Mathematics Subject Classification. Primary 14-02, 14J70; Secondary 14M20, 14E08, $14 \mathrm{~N} 25$.

Partial financial support was provided by the NSF under grant number DMS-1362960 and by the Nemmers Prize of Northwestern University. 
Conceptually, the simplest algebraic sets are hypersurfaces; these are defined by one equation. That is, an affine algebraic hypersurface of dimension $n$ is the zero set of a polynomial $h\left(x_{1}, \ldots, x_{n+1}\right)$ :

$$
X=X(h):=\left(h\left(x_{1}, \ldots, x_{n+1}\right)=0\right) \subset \mathbb{A}^{n+1} .
$$

We say that $X$ is irreducible if $h$ is irreducible, and we call deg $h$ the degree of $X$.

Working over $\mathbb{C}$, one can (almost) harmlessly identify the algebraic hypersurface $X$ with the corresponding subset of $\mathbb{C}^{n+1}$, but already over $\mathbb{R}$ we have to be more careful. For example, $x_{1}^{2}+x_{2}^{2}+1$ has no real zeros. Thus when we say "algebraic hypersurface over $\mathbb{R}$ ", we think of not just the real zero set but also the complex zero set. (One could also think about zero sets in even larger fields, for example $\mathbb{C}\left(t_{1}, \ldots, t_{m}\right)$, but it turns out that knowing the zero set over $\mathbb{C}$, or over any algebraically closed field, determines the zero sets over any larger field.)

Thus in practice we usually think of an affine hypersurface $X=X(h)$ as a subset of $\mathbb{C}^{n+1}$, and we keep in mind that it may have been defined by an equation whose coefficients are real or rational. We use $X(\mathbb{C})$ to denote the set of complex solutions of the equation $h(\mathbf{x})=0$. If $h$ has real or rational coefficients, then it also makes sense to ask about the real solutions $X(\mathbb{R})$ or rational solutions $X(\mathbb{Q})$.

We say that two hypersurfaces are linearly isomorphic if they differ only by a linear change of coordinates. In principle one can always decide whether two hypersurfaces are linearly isomorphic or not, though in practice the computations may be infeasibly long.

The main question we address in these notes is whether there are other, nonlinear maps and isomorphisms between algebraic hypersurfaces. The problem naturally divides into two topics.

Question 1. When are two algebraic hypersurfaces $X_{1}$ and $X_{2}$ isomorphic?

Question 2. When are two algebraic hypersurfaces $X_{1}$ and $X_{2}$ "almost" isomorphic?

Note that we have not yet defined what an "isomorphism" is and gave yet no hint what an "almost" isomorphism should be. Before we give these in Definition 13 . we start with an example in Section 1 .

Prerequisites. This article is aimed at readers with no prior knowledge of algebraic geometry. We focus on concrete examples that can be worked out by hand yet give a good illustration of the general principles. Thus we also avoid any uses of commutative algebra and of several complex variables, two topics that usually undergird algebraic geometry. The usual (topological) cohomology groups make a fleeting appearance in Section 6 and differential forms are used in Section 7 .

However, the discussions of Section 10 need some concepts of algebraic geometry, mainly ampleness and the canonical class.

Throughout, we work with polynomials with complex coefficients and their complex solutions. For most claims this is not very important, but in some examples it is quite interesting to know what happens when we focus on rational solutions; this is the theory of Diophantine equations. We point out these, and connections with other topics, in Side Remarks. These can be skipped.

Algebraic geometers almost always work with systems of equations, leading to the notions of algebraic sets and varieties. For most of the results that we discuss, 
these form the natural setting; see [Sha74, Mum76, Rei88, CLO92, Dol12, EH16] for introductions.

However, many definitions and results are much easier to state for hypersurfaces, and, once the foundational material is well understood, the basic difficulties are usually very similar.

\section{Stereographic Projection}

The oldest example of an "almost" isomorphism between hypersurfaces is the stereographic projection. It may well have been invented-originally under the name planisphaerium - to create star charts that represent the celestial sphere in a plane. It has been used in mapmaking since the sixteenth century.

A theorem that Ptolemy attributes to Hipparchus ( 190-120 BC) says that stereographic projection maps circles to circles. Halley (best known for his comet) proved in Hal1695] that stereographic projection also preserves angles. Now we refer to these properties by saying that stereographic projection is conformal.

Instead of these metric properties, we are more interested in the actual formulas that define it.

3. Stereographic projection. The formulas for stereographic projection are nicest if we project the unit sphere

$$
\mathbb{S}^{n}:=\left(x_{1}^{2}+\cdots+x_{n+1}^{2}=1\right) \subset \mathbb{R}^{n+1}
$$

from the south pole $\mathbf{p}_{0}:=(0, \ldots, 0,-1)$ to the plane $x_{n+1}=0$ where we use $y_{1}, \ldots, y_{n}$ as coordinates instead of $x_{1}, \ldots, x_{n}$,

Geometrically, pick any point $\mathbf{p} \in \mathbb{S}^{n}$ (other than the south pole), and let $\pi(\mathbf{p})$ denote the intersection point of the line $\left\langle\mathbf{p}_{0}, \mathbf{p}\right\rangle$ with the hyperplane $\left(x_{n+1}=0\right)$.

Algebraically, we get that

$$
\pi\left(x_{1}, \ldots, x_{n+1}\right)=\left(\frac{x_{1}}{1+x_{n+1}}, \ldots, \frac{x_{n}}{1+x_{n+1}}\right)
$$

with inverse

$$
\pi^{-1}\left(y_{1}, \ldots, y_{n}\right)=\left(\frac{2 y_{1}}{1+\Sigma}, \ldots, \frac{2 y_{n}}{1+\Sigma}, \frac{1-\Sigma}{1+\Sigma}\right)
$$

where $\Sigma=y_{1}^{2}+\cdots+y_{n}^{2}$.

Here $\pi$ is not defined at the points where $x_{n+1}=-1$, and $\pi^{-1}$ is not defined at the points where $y_{1}^{2}+\cdots+y_{n}^{2}=-1$. In the real case we get a one-to-one map $\mathbb{S}^{n} \backslash$ (south pole) $\cong \mathbb{R}^{n}$ and both $\pi$ and $\pi^{-1}$ are given by rational functions.

However, we know that we should also look at the complex case. Note that

$$
\mathbb{S}^{n}(\mathbb{C}):=\left(x_{1}^{2}+\cdots+x_{n+1}^{2}=1\right) \subset \mathbb{C}^{n+1}
$$

is not a sphere, it is not even compact. Both indeterminacy sets

$$
\mathbb{S}^{n}(\mathbb{C}) \cap\left(x_{n+1}=-1\right) \text { and }\left(y_{1}^{2}+\cdots+y_{n}^{2}=-1\right)
$$

are $(n-1)$-dimensional hypersurfaces. We get a one-to-one map between the sets

$$
\mathbb{S}^{n}(\mathbb{C}) \backslash\left(x_{n+1}=-1\right) \text { and } \mathbb{C}^{n} \backslash\left(y_{1}^{2}+\cdots+y_{n}^{2}=-1\right),
$$

which is given by rational functions in both directions.

This will be our definition of a birational map, which is the usual name for the almost isomorphism of Question 2. 
A number theoretic variant of stereographic projection is the following.

4. Side Remark: A diophantine equation. Consider the diophantine equation

$$
a_{1} x_{1}^{2}+\cdots+a_{n} x_{n}^{2}+x_{n+1}^{2}=1 \quad \text { where } \quad a_{i} \in \mathbb{Q} .
$$

It determines the quadric hypersurface

$$
Q^{n}:=\left(a_{1} x_{1}^{2}+\cdots+a_{n} x_{n}^{2}+x_{n+1}^{2}=1\right) .
$$

Again projecting from the south pole $\mathbf{p}_{0}:=(0, \ldots, 0,-1)$, we get the formulas

$$
\pi\left(x_{1}, \ldots, x_{n+1}\right)=\left(\frac{x_{1}}{1+x_{n+1}}, \ldots, \frac{x_{n}}{1+x_{n+1}}\right)
$$

with inverse

$$
\pi^{-1}\left(y_{1}, \ldots, y_{n}\right)=\left(\frac{2 y_{1}}{1+\Sigma}, \ldots, \frac{2 y_{n}}{1+\Sigma}, \frac{1-\Sigma}{1+\Sigma}\right),
$$

where now $\Sigma=a_{1} y_{1}^{2}+\cdots+a_{n} y_{n}^{2}$.

Thus, we conclude that all rational solutions of equation (4.1) satisfying $x_{n+1} \neq$ -1 can be written as in (4.4) for rational numbers $y_{1}, \ldots, y_{n}$.

Note that in (4.1) we are working with the special case when $a_{n+1}=1$. We could allow instead $a_{n+1}$ to be arbitrary, but then we would need to project from the point $\left(0, \ldots, 0,-\sqrt{a_{n+1}}\right)$. If $a_{n+1}$ is not a square, the resulting formulas give irrational solutions of the equation. See Theorem 7 for the right generalization.

\section{Projective hypersurfaces}

An affine hypersurface of dimension $\geq 1$ is never compact over $\mathbb{C}$, thus we frequently work with its closure in $\mathbb{C P}^{n+1}$.

Definition 5 (Projective space). We will think of the points of $\mathbb{C P}^{n+1}$ as the lines through the origin in $\mathbb{C}^{n+2}$. Thus we use homogeneous coordinates $\left(X_{0}: \cdots: X_{n+1}\right)$, where at least one of the $X_{i}$ must be nonzero. Furthermore,

$$
\left(\lambda X_{0}: \cdots: \lambda X_{n+1}\right)=\left(X_{0}: \cdots: X_{n+1}\right) \text { for all } \lambda \in \mathbb{C}^{*} .
$$

A polynomial $h\left(X_{0}, \ldots, X_{n+1}\right)$ cannot be evaluated at a point of $\mathbb{C P}^{n+1}$ since usually $h\left(\lambda X_{0}: \cdots: \lambda X_{n+1}\right) \neq h\left(X_{0}: \cdots: X_{n+1}\right)$. However, if $H$ is homogeneous of degree $d$, then

$$
H\left(\lambda X_{0}: \cdots: \lambda X_{n+1}\right)=\lambda^{d} H\left(X_{0}: \cdots: X_{n+1}\right),
$$

thus the zero set of a homogeneous polynomial is well defined. Thus, we get projective hypersurfaces

$$
X=X(H):=\left(H\left(X_{0}: \cdots: X_{n+1}\right)=0\right) \subset \mathbb{P}^{n+1} .
$$

One can go between the affine and the projective versions by the formulas

$$
\begin{aligned}
h\left(x_{1}, \ldots, x_{n+1}\right) & =H\left(1, x_{1}, \ldots, x_{n+1}\right) \text { and } \\
H\left(X_{0}, \ldots, X_{n+1}\right) & =X_{0}^{\operatorname{deg} h} \cdot h\left(\frac{X_{1}}{X_{0}}, \ldots, \frac{X_{n+1}}{X_{0}}\right) .
\end{aligned}
$$

Of course any of the $X_{i}$ could play the special role of $X_{0}$ above, so we usually think of $\mathbb{C P}^{n+1}$ as being covered by $n+2$ charts, each isomorphic to $\mathbb{C}^{n+1}$.

The cone over $X$ is the affine hypersurface

$$
C_{X}=C_{X(H)}:=\left(H\left(X_{0}, \ldots, X_{n+1}\right)=0\right) \subset \mathbb{A}^{n+2} .
$$

This gives another way to go between projective and affine questions. 
Note. It would be quite convenient to keep the notational distinction between affine coordinates $x_{i}$ and projective coordinates $X_{i}$, but people usually use lower case $x_{i}$ to denote both affine and projective coordinates.

Modern algebraic geometry usually considers the projective variant the basic object and the affine versions as the local charts on the projective hypersurface. Note, however, that in algebraic geometry the local charts are very big; they are always dense in the corresponding projective hypersurface.

Definition 6. A point $\mathbf{p} \in X(G)$ on a projective hypersurface is called smooth if $\frac{\partial G}{\partial x_{i}}(\mathbf{p}) \neq 0$ for some $i$. If we are over $\mathbb{C}$ or $\mathbb{R}$, the implicit function therem tells us that $X$ is an $n$-dimensional submanifold of $\mathbb{C P}^{n+1}$ or of $\mathbb{R} \mathbb{P}^{n+1}$ at its smooth points. Here of course over $\mathbb{C}$ we count complex dimension, which is twice the real dimension.

The tangent plane $T_{\mathbf{p}} X$ (viewed as a hyperplane in $\mathbb{P}^{n+1}$ ) at a smooth point $\mathbf{p}:=\left(p_{0}: \cdots: p_{n+1}\right)$ is given by the equation

$$
\sum_{i} \frac{\partial G}{\partial x_{i}}(\mathbf{p}) \cdot x_{i}=0 .
$$

(Our first inclination would be write $\sum_{i} \frac{\partial G}{\partial x_{i}}(\mathbf{p}) \cdot\left(x_{i}-p_{i}\right)=0$ instead. This does not seem to make sense as an equation since it is not homogeneous. Luckily,

$$
\sum_{i} \frac{\partial G}{\partial x_{i}}(\mathbf{p}) \cdot p_{i}=\operatorname{deg} G \cdot G(\mathbf{p})=0
$$

since $G$ is homogeneous, thus, after all, we do get (6, 1) this way.)

The other points are singular. Thus the set of all singular points is defined by the equations

$$
\frac{\partial G}{\partial x_{0}}=\cdots=\frac{\partial G}{\partial x_{n+1}}=0 .
$$

It is thus a closed set which is easily seen to be nowhere dense if $G$ is irreducible.

We have the following rather straightforward generalization of the formulas (3,2)(3).3) for the stereographic projection.

Theorem 7. Consider the irreducible quadric hypersurface

$$
Q^{n}:=\left(G\left(x_{0}, \ldots, x_{n+1}\right)=0\right) \subset \mathbb{P}^{n+1} .
$$

Let $\mathbf{p} \in Q^{n}$ be a smooth point with tangent plane $T_{\mathbf{p}} Q$. Pick any hyperplane $H \cong \mathbb{P}^{n}$ that does not contain $\mathbf{p}$. Then the following hold.

(1) Projection of $\mathbb{P}^{n+1}$ from $\mathbf{p}$ to $H$ gives a one-to-one map

$$
\pi: Q^{n} \backslash T_{\mathbf{p}} Q \longrightarrow H \backslash T_{\mathbf{p}} Q \cong \mathbb{A}^{n} .
$$

(2) The coordinate functions of $\pi$ are quotients of linear polynomials.

(3) The coordinate functions of $\pi^{-1}$ are quotients of quadratic polynomials.

Side Remark. Furthermore, if the coefficients of $G$, of the equation of $H$, and the coordinates of $\mathbf{p}$ are all in a field $k$, then so are the coefficients of $\pi$ and $\pi^{-1}$. 


\section{RATiOnAL AND BIRATIONAL MAPS}

Definition 8 (Rational maps, affine case). The basic functions in algebraic geometry are polynomials $p\left(x_{1}, \ldots, x_{n}\right)$ and their quotients

$$
\phi\left(x_{1}, \ldots, x_{n}\right)=\frac{p\left(x_{1}, \ldots, x_{n}\right)}{q\left(x_{1}, \ldots, x_{n}\right)}
$$

are called rational functions. Since a polynomial ring has unique factorization, we may assume that $p, q$ are relatively prime. In this case $\phi$ is not defined along the hypersurface $(q=0)$.

A rational map from $\mathbb{A}^{n}$ to $\mathbb{A}^{m}$ is a map given by rational coordinate functions

$$
\Phi\left(x_{1}, \ldots, x_{n}\right)=\left(\phi_{1}\left(x_{1}, \ldots, x_{n}\right), \ldots, \phi_{m}\left(x_{1}, \ldots, x_{n}\right)\right),
$$

where $\phi_{i}=p_{i} / q_{i}$. As before, $\Phi$ need not be everywhere defined.

Definition 9 (Rational maps, projective case). As we already noted in (5,2), a polynomial or a rational function $p\left(x_{0}, \ldots, x_{n}\right)$ usually cannot be evaluated at a point of $\mathbb{P}^{n}$. However, if $\phi$ is a quotient of two homogeneous polynomials of the same degree, then

$$
\phi\left(\lambda x_{0}: \ldots: \lambda x_{n}\right)=\phi\left(x_{0}: \cdots: x_{n}\right)
$$

and $\phi$ defines a rational function on $\mathbb{P}^{n}$.

A rational map from $\mathbb{P}^{n}$ to $\mathbb{P}^{m}$ is a map given by rational functions

$$
\Phi\left(x_{0}: \cdots: x_{n}\right)=\left(\phi_{0}\left(x_{0}: \cdots: x_{n}\right): \cdots: \phi_{m}\left(x_{0}: \cdots: x_{n}\right)\right) .
$$

We denote rational maps by a dashed arrow $\rightarrow-\rightarrow$.

The nonuniqueness of projective coordinates leads to two basic questions: When are two rational maps the "same", and where is a rational map defined?

Given two rational maps $\Phi=\left(\phi_{0}, \ldots, \phi_{m}\right)$ and $\Phi^{\prime}=\left(\phi_{0}^{\prime}, \ldots, \phi_{m}^{\prime}\right)$, they agree at a point $\mathbf{x}$ iff

$$
\left(\phi_{0}(\mathbf{x}): \cdots: \phi_{m}(\mathbf{x})\right)=\lambda \cdot\left(\phi_{0}^{\prime}(\mathbf{x}): \cdots: \phi_{m}^{\prime}(\mathbf{x})\right)
$$

for some $\lambda \neq 0$. Here $\lambda$ itself can depend on $\mathbf{x}$, thus it is a rational function $\lambda(\mathbf{x})$. Thus, two rational maps $\Phi$ and $\Phi^{\prime}$ are considered the same if

$$
\phi_{0} / \phi_{0}^{\prime}=\cdots=\phi_{m} / \phi_{m}^{\prime} .
$$

Next write each $\phi_{i}$ is a quotient of two polynomials $\phi_{i}=p_{i} / q_{i}$. This suggests that $\phi_{i}$ is not be defined along the hypersurfaces $\left(q_{i}=0\right)$. Furthermore, every point in $\mathbb{P}^{m}$ has at least one nonzero coordinate, so $\Phi$ is also not defined along the intersection of the hypersurfaces $\left(p_{0}=0\right) \cap \cdots \cap\left(p_{n}=0\right)$. Do we get the same answer if we start with $\Phi^{\prime}$ ? Confusingly, the answer is no, but we can turn the nonuniqueness of projective coordinates to our advantage. Multiplying through with the least common denominator of the $\phi_{i}$ in (9,2) and canceling common factors, we can represent $\Phi$ as

$$
\Phi(\mathbf{x})=\left(P_{0}(\mathbf{x}): \cdots: P_{m}(\mathbf{x})\right),
$$

where the $P_{i}$ are polynomials that do not have a common factor. Such a representation of a rational map $\mathbb{P}^{n} \rightarrow \mathbb{P}^{m}$ is unique (up to multiplication by a constant), and $\Phi$ is defined outside the common zero set of the $P_{i}$. From this we can read off two important properties. 
Claim 9,6. $\Phi: \mathbb{P}^{n} \rightarrow \mathbb{P}^{m}$ is everywhere defined iff the $P_{i}$ in $(9,5)$ have no common zero.

Claim 9.7. Write $P_{i}(\mathbf{x})=c_{i} \prod_{j} r_{j}(\mathbf{x})^{m(i, j)}$, where the $c_{i}$ are constants, the $r_{j}$ are irreducible polynomials, and $m(i, j) \geq 0$. Then $\Phi$ is defined outside the set

$$
Z:=\bigcup_{j_{1} \neq j_{2}}\left(r_{j_{1}}(\mathbf{x})=r_{j_{2}}(\mathbf{x})=0\right)
$$

Note that $Z$ is made up of many pieces, but each of them is defined by the vanishing of two relatively prime polynomials. Thus we expect that $Z$ has codimension $\geq 2$ in $\mathbb{P}^{n}$. This is not hard to see; we state a general version as Theorem 12 .

Definition 10 (Rational maps of hypersurfaces). A rational function on a hypersurface $X \subset \mathbb{P}^{n+1}$ is the restriction of a rational function $\phi$ on $\mathbb{P}^{n+1}$ to $X$, provided the restriction makes sense. That is, when $\phi$ is defined on a dense open subset of $X$.

As before, a rational map $\Phi$ from a hypersurface $X \subset \mathbb{P}^{n+1}$ to $\mathbb{P}^{m}$ is given by rational functions $\left(\phi_{0}: \cdots: \phi_{m}\right)$. If $\Phi(X)$ is contained in a hypersurface $Y \subset \mathbb{P}^{m}$, then $\Phi$ defines a rational map $\Phi: X \rightarrow Y$. (If we work over $\mathbb{C}$, then by $\Phi(X) \subset Y$ we mean that $\Phi(\mathbf{p}) \in Y$ whenever $\Phi$ is defined at $\mathbf{p} \in X$. In general we need the algebraic version given in Remark 15.)

We use three important basic properties of rational functions.

Claim 10.1. If $X$ is irreducible and $\phi$ is defined at a single point of $X$, then it is defined on a dense open subset of $X$, cf. Sha74, Sec.I.3.2].

Claim 10,2. If $X \subset \mathbb{A}^{n+1}$ is affine and $\left.\phi\right|_{X}$ is everywhere defined, then there is a polynomial $p$ on $\mathbb{A}^{n+1}$ such that $\left.p\right|_{X}=\left.\phi\right|_{X}$. For example, $\frac{x^{3}-y}{x+y}$ has poles but its restriction to the $x$-axis is everywhere defined. We can take $p:=x^{2}$.

This is usually proved as a consequence of Hilbert's Nullstellensatz, see Sha74, Sec.I.3.2].

Purity Principle 10.3. Let $\phi: X \rightarrow \mathbb{P}^{1}$ be a rational function on a hypersurface $X$. Then the set of points where $\phi$ is not defined has codimension 1 everywhere.

This is more subtle. In books it is usually treated as a combination of Krull's principal ideal theorem and Serre's $S_{2}$ property for hypersurfaces; however, this form goes back to Macaulay [Mac1916].

Side Remark. Readers may recognize Purity Principle 10,3 as an algebraic counterpart of Hartogs's extension theorem in several complex variables.

Definition 11 (Morphisms). $\Phi$ is defined at a point $\mathbf{p} \in X$ if it has some representation

$$
\Phi(\mathbf{x})=\left(\phi_{0}(\mathbf{x}): \cdots: \phi_{m}(\mathbf{x})\right)
$$

as in (9,2), where every coordinate function is defined at $\mathbf{p}$ and at least one of them is nonzero. (The traditional terminology is " $\Phi$ is regular" at $\mathbf{p}$, but be warned that "regular" has other, conflicting uses, even in algebraic geometry.)

A morphism is a rational map that is everywhere defined. We denote morphisms by a solid arrow $\rightarrow$.

Warning 11,2. A major difficulty is that, unlike for $\mathbb{P}^{n}$ where we could write down the optimal representation in $(9,5)$, there need not be a single form (11,1) that shows regularity at every point. 
For example, consider the smooth hypersurface $Q:=\left(x_{1} x_{2}=x_{3} x_{4}\right) \subset \mathbb{P}^{3}$ and the rational map

$$
\Phi: Q \rightarrow \mathbb{P}^{1} \text { given by }\left(x_{1}: x_{2}: x_{3}: x_{4}\right) \mapsto\left(x_{1}: x_{3}\right) .
$$

It is defined outside $\left(x_{1}=x_{3}=0\right)$. Next note that $x_{1} / x_{4}=x_{3} / x_{2}$, thus $\Phi$ can also be written as

$$
\Phi:\left(x_{1}: x_{2}: x_{3}: x_{4}\right) \mapsto\left(x_{4}: x_{2}\right),
$$

hence $\Phi$ is defined outside $\left(x_{4}=x_{2}=0\right)$. Thus $\Phi$ is everywhere defined on $Q$-it is a morphism.

However, no matter how we write $\Phi=\left(p_{1}: p_{2}\right)$ for some polynomials $p_{i}$, the system $p_{1}=p_{2}=x_{1} x_{2}-x_{3} x_{4}=0$ always has a solution in $\mathbb{P}^{3}$. Thus, unlike Claim 96 , we cannot conclude that $\Phi$ is everywhere defined using just one representation of it.

A major theorem of Lefschetz implies that the situation is better for smooth hypersurfaces of dimension $\geq 3$; see paragraph 34 for a discussion.

By contrast, the following generalization of (96) holds; see [Sha74, Sec.II.3.1].

Theorem 12. Let $X$ be a smooth hypersurface, and let $\Phi: X \rightarrow \mathbb{P}^{m}$ be a rational map. Then there is a closed subset $Z \subset X$ of codimension $\geq 2$ such that $\Phi$ is defined on $X \backslash Z$.

Now we come to the definition of "isomorphism" and "almost isomorphism."

Definition 13. Let $\Phi: X \rightarrow Y$ be a rational map between hypersurfaces.

(1) $\Phi$ is birational if there is a rational map $\Phi^{-1}: Y \rightarrow X$ that is the inverse of $\Phi$. That is, $\Phi^{-1} \circ \Phi$ and $\Phi \circ \Phi^{-1}$ are both the identity, wherever they are defined. If this holds, then we say that $X$ and $Y$ are birationally equivalent or birational.

(2) An $n$-dimensional hypersurface $X$ is called rational if it is birational to $\mathbb{P}^{n}$.

(3) $\Phi$ is an isomorphism if both $\Phi$ and $\Phi^{-1}$ are morphisms, that is, everywhere defined.

14. Why birational equivalence? The notion of birational equivalence usually seems unnatural to people coming from topology - where open dense subsets remember only the dimension of the manifold - but it should be more natural to people who work in analysis, measure theory, or probability, where ignoring measure 0 subsets is common in many questions.

Algebraic geometers feel that this concept is forced upon us, since many interesting maps, for example the stereographic projection, are not defined everywhere. Thus with many questions it is profitable to study first its birational version, and, once that is understood, try to see whether the question makes sense or is solvable up to isomorphism.

Remark 15. Note that an affine hypersurface is birational to its projective closure, thus its birationality can be checked using affine equations. Two hypersurfaces

$$
\begin{aligned}
X & :=\left(h\left(x_{1}, \ldots, x_{n+1}\right)=0\right) \subset \mathbb{A}_{\mathbf{x}}^{n+1} \\
Y & :=\left(g\left(y_{1}, \ldots, y_{n+1}\right)=0\right) \subset \mathbb{A}_{\mathbf{y}}^{n+1}
\end{aligned} \text { and }
$$

are birational if there are rational maps

$$
\begin{aligned}
& \Phi=\left(\phi_{1}\left(x_{1}, \ldots, x_{n+1}\right), \ldots, \phi_{n+1}\left(x_{1}, \ldots, x_{n+1}\right)\right) \quad \text { and } \\
& \Psi=\left(\psi_{1}\left(y_{1}, \ldots, y_{n+1}\right), \ldots, \psi_{n+1}\left(y_{1}, \ldots, y_{n+1}\right)\right)
\end{aligned}
$$


with the following properties.

[15.1) $\Phi$ maps $X$ to $Y$. In terms of equations this means that $g\left(\phi_{1}, \ldots, \phi_{n+1}\right)$ vanishes on $X$. That is, if we write $g\left(\phi_{1}, \ldots, \phi_{n+1}\right)$ as the quotient of two relatively prime polynomials, then the numerator is divisible by $h$.

(15)2) $\Psi$ maps $Y$ to $X$.

(15]3) $\Phi: X \rightarrow Y$ and $\Psi: Y \rightarrow X$ are inverses of each other. Note that

$$
\Psi \circ \Phi=\left(\psi_{1}\left(\phi_{1}, \ldots, \phi_{n+1}\right), \ldots, \psi_{n+1}\left(\phi_{1}, \ldots, \phi_{n+1}\right)\right),
$$

and this is the identity on $X$ iff $x_{i}-\psi_{i}\left(\phi_{1}, \ldots, \phi_{n+1}\right)$ vanishes on $X$ for every $i$. Similarly, $y_{j}-\phi_{j}\left(\psi_{1}, \ldots, \psi_{n+1}\right)$ vanishes on $Y$ for every $j$.

\section{The MAIN QUeSTIONS}

Now we are ready to formulate the precise versions of Questions 1 and 2 In both cases the ideal complete answer would consists of two steps.

- Describe a set of elementary isomorphisms/birational maps between hypersurfaces.

- Prove that every isomorphism/birational map between hypersurfaces is elementary, or at least a composite of elementary maps.

So what are these elementary isomorphisms/birational maps?

Isomorphisms of projective hypersurfaces. For projective hypersurfaces $X \subset$ $\mathbb{P}^{n+1}$ the elementary isomorphisms are those that are induced by an automorphism of $\mathbb{P}^{n+1}$. It is not hard to see that $\operatorname{Aut}\left(\mathbb{P}^{n+1}\right) \cong \mathrm{PGL}_{n+2}$ (cf. [Sha74, III.1.Exrc.17]), so these are exactly the linear isomorphisms. This leads to the projective version of Question 1 .

Question 16. Let $X_{1}, X_{2}$ be projective algebraic hypersurfaces in $\mathbb{P}^{n+1}$, and let $\Phi: X_{1} \rightarrow X_{2}$ be an isomorphism. Is $\Phi$ linear? That is, is there an automorphism $\Psi \in \operatorname{Aut}\left(\mathbb{P}^{n+1}\right) \cong \mathrm{PGL}_{n+2}$ such that $\Phi=\left.\Psi\right|_{X_{1}}$ ?

We discuss almost complete answers to this is Section 6 .

Isomorphisms of affine hypersurfaces. The projective case suggests that for affine hypersurfaces the elementary isomorphisms should be those that are induced by an automorphism of $\mathbb{A}^{n+1}$. A major difficulty is that $\operatorname{Aut}\left(\mathbb{A}^{n+1}\right)$ is infinite dimensional. For example, given any polynomials $g_{i}\left(x_{i+1}, \ldots, x_{n+1}\right)$, the map

$$
\Phi:\left(x_{1}, \ldots, x_{n+1}\right) \mapsto\left(x_{1}+g_{1}, x_{2}+g_{2}, \ldots, x_{n+1}+g_{n+1}\right)
$$

is an automorphism of $\mathbb{A}^{n+1}$. These automorphisms and $\mathrm{GL}_{n}$ together generate the tame subgroup of $\operatorname{Aut}\left(\mathbb{A}^{n+1}\right)$. If $n=1$, then we get the whole $\operatorname{Aut}\left(\mathbb{A}^{2}\right)$ by $\operatorname{Abh} 77$. but the analogous result does not hold in higher dimensions. The counterexample

$$
(x, y, z) \mapsto\left(x-2\left(x z+y^{2}\right) y-\left(x z+y^{2}\right)^{2} z, y+\left(x z+y^{2}\right) z, z\right)
$$

was proposed by Nagata Nag72 and proved in [SU04] see Kur16] for a survey and AL92 for a complex analytic version.

Already, the simplest case of the affine isomorphism problem is a quite nontrivial result of [AM75] and [Suz74].

Theorem 17 (Abhyankar, Moh, and Suzuki). Let $C \subset \mathbb{A}^{2}$ be a curve, and let $\Phi: C \rightarrow \mathbb{A}^{1}$ be an isomorphism. Then $\Phi$ extends to an isomorphism

$$
\Psi:\left(C \subset \mathbb{A}^{2}\right) \cong\left((\text { coordinate axis }) \subset \mathbb{A}^{2}\right) .
$$


The higher dimensional generalization of this is the Abhyankar-Sathaye conjecture (about which very little is known) and a series of counterexamples is proposed in [CD94; see also vdE00, Chap.5].

Conjecture 18. Let $X \subset \mathbb{A}^{n+1}$ be a hypersurface, and let $\Phi: X \rightarrow \mathbb{A}^{n}$ be an isomorphism. Then $\Phi$ extends to an isomorphism

$$
\Psi:\left(X \subset \mathbb{A}^{n+1}\right) \cong\left((\text { coordinate hyperplane }) \subset \mathbb{A}^{n+1}\right) .
$$

Interestingly, the union of the $n$ coordinate hyperplanes $\left(x_{1} \cdots x_{n}=0\right)$ has a unique embedding into $\mathbb{C}^{n}$ by [Jel97]. For some recent results connecting the affine isomorphism problem with the methods of Section 9] see [CDP17.

Birational equivalence of hypersurfaces. It seems natural to formulate Question 2 in two variants. Note that an affine hypersurface is birational to its projective closure, so for the birationality questions there is no need to distinguish the affine and the projective cases.

Question 19. Which algebraic hypersurfaces are rational (as in Definition $13(2))$ ?

Question 20. When are two algebraic hypersurfaces birational to each other?

Example 21 (Rational hypersurfaces). It has been long understood that even high degree hypersurfaces can be rational if they are very singular. Here are some examples of this.

(21, 1) Let $X \subset \mathbb{P}^{n+1}$ be given by an equation

$$
H_{d-1}\left(x_{0}, \ldots, x_{n}\right) x_{n+1}+H_{d}\left(x_{0}, \ldots, x_{n}\right)=0,
$$

where $H_{j}$ is homogeneous of degree $j$. Projection from the point $(0: \cdots: 0: 1)$ to the hyperplane $\left(x_{n+1}=0\right)$ is birational, the inverse is given by

$$
\left(x_{0}: \cdots: x_{n}\right) \mapsto\left(x_{0}: \cdots: x_{n}: \frac{H_{d}\left(x_{0}, \ldots, x_{n}\right)}{H_{d-1}\left(x_{0}, \ldots, x_{n}\right)}\right) .
$$

If $d=2$, then this is the stereographic projection. If $d \geq 3$, then $(0: \cdots: 0: 1)$ is a singular point of $X$.

[21.2) Let $X \subset \mathbb{P}^{2 n+1}$ be a hypersurface of degree $2 d+1$ given by an equation of the form

$$
\sum_{I, J, k} a_{I, J, k} \cdot M_{I} \cdot N_{J} \cdot x_{k}
$$

where $M_{I}$ is a degree $d$ monomial in the variables $x_{0}, \ldots, x_{n}$ and $N_{J}$ is a degree $d$ monomial in the variables $x_{n+1}, \ldots, x_{2 n+1}$ and $x_{k}$ is arbitrary. (In particular, $X$ contains the linear subspaces $L_{1}:=\left(x_{0}=\cdots=x_{n}=0\right)$ and $L_{2}:=\left(x_{n+1}=\cdots=x_{2 n+1}=0\right)$.) Then $X$ is rational. We will work out the $2 d+1=3$ case in detail in Proposition 24 .

If $2 d+1=3$, then a general such $X$ is smooth, but for $2 d+1 \geq 5$ it is always singular along the $L_{i}$.

(21)3) Take degree $d$ homogeneous polynomials $p_{0}(\mathbf{x}), \ldots, p_{n+1}(\mathbf{x})$. They define a rational map $\Phi: \mathbb{P}^{n} \rightarrow \mathbb{P}^{n+1}$. It is not hard to show that if the $p_{i}$ are general, then $\Phi$ is a morphism whose image $Y=Y\left(p_{0}, \ldots, p_{n+1}\right)$ is a rational hypersurface of degree $d^{n}$. However, $Y$ has very complicated self-intersections. 
These and many other examples suggest that Questions 19 and 20 are reasonable only if the hypersurfaces are smooth or mildly singular. We will focus on the smooth cases.

Question 22. Which smooth algebraic hypersurfaces are rational?

Question 23. When are two smooth algebraic hypersurfaces birational to each other?

So far is not even clear that not all hypersurfaces are rational. We see later that this is not at all the case, but first we give some rational examples.

\section{Rationality of CUBiC hypersurfaces}

We proved in Theorem 7 that a smooth quadric hypersurface is rational. In this section we consider the rationality of cubic hypersurfaces. This is a topic with many interesting results and still unsolved questions. The simplest rationality construction is the following special case of (21,2).

Proposition 24. Let $S \subset \mathbb{P}^{3}$ be a cubic surface that contains two disjoint lines. Then $S$ is rational.

Geometric Proof. Let the lines be $L_{1}, L_{2}$. Pick points $\mathbf{p}_{i} \in L_{i}$. The line connecting them, which we call $\left\langle\mathbf{p}_{1}, \mathbf{p}_{2}\right\rangle$, meets $S$ in three points. We already know two of them, namely $\mathbf{p}_{1}, \mathbf{p}_{2}$. Let $\phi\left(\mathbf{p}_{1}, \mathbf{p}_{2}\right)$ be the third intersection point. This gives a rational map

$$
\phi: L_{1} \times L_{2} \rightarrow S \text {. }
$$

To get its inverse, pick $\mathbf{q} \in \mathbb{P}^{3}$ and let $\pi: \mathbb{P}^{3} \rightarrow \mathbb{P}^{2}$ denote the projection from $\mathbf{q}$. Then $\pi\left(L_{1}\right), \pi\left(L_{2}\right)$ are two lines in $\mathbb{P}^{2}$, hence they meet at a unique point $\mathbf{q}^{\prime}$. Thus we get $\mathbf{p}_{i} \in L_{i}$ such that $\pi\left(\mathbf{p}_{i}\right)=\mathbf{q}^{\prime}$. This gives

$$
\psi: \mathbb{P}^{3} \rightarrow L_{1} \times L_{2} \text { such that }\left.\psi\right|_{S}=\phi^{-1} .
$$

Algebraic Proof. We can choose coordinates such that $L_{1}=\left(x_{0}=x_{1}=0\right)$ and $L_{2}=\left(x_{2}=x_{3}=0\right)$. Thus the equation of $S$ can be written (nonuniquely) as

$$
\sum_{i=0,1} \sum_{j=2,3} \ell_{i j}(\mathbf{x}) x_{i} x_{j}
$$

where the $\ell_{i j}(\mathbf{x})$ are linear in the variables $x_{0}, \ldots, x_{3}$.

If $p_{1}=\left(a_{0}: a_{1}: 0: 0\right)$ and $p_{2}=\left(0: 0: a_{2}: a_{3}\right)$, then the line connecting them is

$$
\mathbb{P}^{1} \ni(s: t) \mapsto\left(s a_{0}: s a_{1}: t a_{2}: t a_{3}\right) \in \mathbb{P}^{3} .
$$

Substituting into (24)3), we get an equation

$$
\sum_{i=0,1} \sum_{j=2,3} \ell_{i j}\left(s a_{0}: s a_{1}: t a_{2}: t a_{3}\right) s a_{i} t a_{j}=s t \sum_{i=0,1} \sum_{j=2,3} \ell_{i j}\left(s a_{0}: s a_{1}: t a_{2}: t a_{3}\right) a_{i} a_{j} .
$$

After dividing by $s t$, the remaining equation is linear in $s, t$, thus it has a unique solution (up to a multiplicative constant)

(24.6) $(s: t)=\left(-\sum_{i=0,1} \sum_{j=2,3} \ell_{i j}\left(0: 0: a_{2}: a_{3}\right) a_{i} a_{j}: \sum_{i=0,1 j=2,3} \sum_{i j}\left(a_{0}: a_{1}: 0: 0\right) a_{i} a_{j}\right)$.

This can be used to prove the following result of [Cle1866].

Corollary 25 (Clebsch). Every smooth cubic surface over $\mathbb{C}$ is rational. 
Hint of Proof. By Proposition 24 it is enough to find two disjoint lines. In fact, every smooth cubic surface over $\mathbb{C}$ contains 27 lines. This was first proved by Cayley and Salmon in 1849 and published in [Sal1865]; see the next example or any of [Rei88, Chap.7], Rei97, Chap.1], or [Sha74, Sec.IV.2.5] for proofs.

Example 26. Consider the degree $d$ hypersurface

$$
X:=\left(x_{0}^{d}+\cdots+x_{2 n+1}^{d}=0\right) \subset \mathbb{P}^{2 n+1} .
$$

Divide the indices into $n+1$ disjoint ordered pairs $\left(x_{\tau(i)}, x_{\sigma(i)}\right): i=0, \ldots, n$. Fix $d$ th roots $\epsilon_{0}, \ldots, \epsilon_{n}$ of -1 . Then

$$
L=L(\tau, \sigma, \epsilon):=\left(x_{\tau(i)}=\epsilon_{i} x_{\sigma(i)}: i=0, \ldots, n\right)
$$

is a linear space of dimension $n$ contained in $X$. Thus $X$ contains at least

$$
\frac{d^{n+1}(2 n+2) !}{2^{n+1}(n+1) !}
$$

linear subspaces of dimension $n$. Not all but many pairs of these linear subspaces are disjoint, for example $L(\tau, \sigma, \epsilon)$ is disjoint from $L\left(\tau^{\prime}, \sigma^{\prime}, \epsilon^{\prime}\right)$ if $\tau=\tau^{\prime}, \sigma=\sigma^{\prime}$ but $\epsilon_{i} \neq \epsilon_{i}^{\prime}$ for every $i$. For $n=2, d=1$ we get all 27 lines on the particular cubic surface.

The following is proved in [Seg43; see [KSC04, Chap.2] for a modern treatment.

Side Theorem 27 (Segre). The cubic surface

$$
S=S\left(a_{0}, a_{1}, a_{2}, a_{3}\right):=\left(a_{0} x_{0}^{3}+a_{1} x_{1}^{3}+a_{2} x_{2}^{3}+a_{3} x_{3}^{3}=0\right) \subset \mathbb{P}^{3}
$$

defined over a field $k$ is not rational over $k$ if for every permutation $\sigma$ of the indices, the quotient $\left(a_{\sigma(0)} a_{\sigma(1)}\right) /\left(a_{\sigma(2)} a_{\sigma(3)}\right)$ is not a cube in $k$.

28. Cubic 3-folds. In contrast to Corollary 25], smooth cubic 3-folds $X_{3} \subset \mathbb{P}^{4}$ are all nonrational CG72. This is proved by analyzing the Hodge structure of the cohomology groups. This method has been very successful for many other threedimensional cases, but extensions to higher dimensions are still lacking.

29. Cubic 4-folds. A cubic 4-fold is defined by a degree 3 homogeneous polynomial in six variables. These form a vector space of dimension $\left(\begin{array}{l}8 \\ 5\end{array}\right)=56$. Since we do not care about multiplicative constants, we can think of the set of all cubic 4-folds as points in a $\mathbb{P}^{55}$. The smooth ones correspond to an open subset of it. (This is a general feature of algebraic geometry. Isomorphism classes frequently naturally correspond to points of another algebraic variety, called the moduli space.)

A long-standing conjecture that grew out of the works of [Mor40 Fan43] says that a general smooth cubic 4-fold is not rational. Has99 describes countably many hypersurfaces $H_{i} \subset \mathbb{P}^{55}$ and conjectures that a cubic 4 -fold $X$ is not rational if the corresponding point $[X] \in \mathbb{P}^{55}$ is outside these hypersurfaces. See the collection BHKV16 for recent surveys.

The strongest results, due to Russo and Staglianò RS17, prove rationality for cubic 4-folds corresponding to three of the hypersurfaces $H_{i}(i=14,26,38$ in Hassett's notation). Just to show that there are some rather subtle rationality constructions, here is one of the beautiful examples they discovered for $H_{38}$.

Pick ten general points $P_{j} \in \mathbb{P}^{2}$ and six general polynomials $p_{i}(x, y, z)$ of degree 10 that vanish with multiplicity 3 at all the points $P_{j}$. Let $F$ be the surface obtained 
as the image of the map

$$
\Psi: \mathbb{P}^{2} \rightarrow \mathbb{P}^{5}:(x: y: z) \mapsto\left(p_{0}(x: y: z): \cdots: p_{5}(x: y: z)\right) .
$$

[RS17] shows that there are five linearly independent quintic polynomials $q_{0}, \ldots, q_{4}$ on $\mathbb{P}^{5}$ that vanish along $F$ with multiplicity 2 . These give a rational map $\Phi: \mathbb{P}^{5}-\rightarrow$ $\mathbb{P}^{4}$ whose general fiber is a conic that intersects $F$ at five points. A conic and a cubic hypersurface typically have six points in common, and $F$ accounts for five of these for the conics of the form $\Phi^{-1}(p)$. Thus, sending a point $p \in \mathbb{P}^{4}$ to the unique sixth intersection point in $X \cap \Phi^{-1}(p)$ gives the inverse of $\left.\Phi\right|_{X}: X \rightarrow \mathbb{P}^{4}$.

It turns out that this construction works for a 54-dimensional subfamily $H_{38}$ of the 55-dimensional family of all cubics.

The method of the proof of these claims in [RS17] shows both the power and the limitations of algebraic geometry. Consider for instance the claim about the five linearly independent quintic polynomials. Standard methods imply that there are at least five such linearly independent quintics. Then other semicontinuity arguments show that if there are exactly five in at least one concrete example, then there are exactly five in almost all cases. Moreover, it is enough to find one such example over a finite field. Russo and Staglianò programmed a computer to try out random cases until it hit one that worked. Since in algebraic geometry the term "almost all" usually means a dense open subset, there is every reason to believe that this leads to a solution.

The disadvantage is that while we proved rationality for smooth cubics corresponding to an open, dense subset in the 54-dimensional hypersurface $H_{38} \subset \mathbb{P}^{55}$, we have no idea which open, dense subset it is.

Fortunately, in this case the recent results of [NS17, KT17] imply that for any family of smooth hypersurfaces parametrized by a variety $V$, once rationality holds for hypersurfaces corresponding to points in an open, dense subset of $V$, then rationality holds for all hypersurfaces in the family, but this requires entirely different methods. In particular, rationality holds for all smooth cubics corresponding to $H_{38}$.

\section{ISOMORPHISM OF HYPERSURFACES}

The following theorem answers Question 16

Theorem 30. Let $X_{1}, X_{2} \subset \mathbb{P}^{n+1}$ be irreducible hypersurfaces, and let $\Phi: X_{1} \cong X_{2}$ be an isomorphism. Then $\Phi$ is obtained by a linear change of coordinates in $\mathbb{P}^{n+1}$, except possibly in the following three cases:

(1) $\operatorname{dim} X_{1}=\operatorname{dim} X_{2}=1$ and $\left\{\operatorname{deg} X_{1}, \operatorname{deg} X_{2}\right\}=\{1,2\}$,

(2) $\operatorname{dim} X_{1}=\operatorname{dim} X_{2}=1$ and $\operatorname{deg} X_{1}=\operatorname{deg} X_{2}=3$, or

(3) $\operatorname{dim} X_{1}=\operatorname{dim} X_{2}=2$ and $\operatorname{deg} X_{1}=\operatorname{deg} X_{2}=4$.

First we describe in detail the exceptional cases.

The first exceptional case is given by the stereographic projection of a plane conic to a line. This is the only dimension where the stereographic projection is an isomorphism.

The second exceptional case is $d=3$ and $n=1$, that is, degree 3 curves in $\mathbb{P}^{2}$. This is the theory of elliptic curves and elliptic integrals. We give a description using the theory of the Weierstrass $\wp$-function; see [Sie69, Chap.1], Sil86, Chap.6], SS03, Chap.9], or many other books on complex analysis for details. 
31. Side Example: Weierstrass $\wp$-function. Let $\Lambda \subset \mathbb{C}$ be a lattice. After multiplying by a suitable $c \in \mathbb{C}^{*}$, we can achieve that its generators are 1 and $\tau$ where $\operatorname{Im}(\tau)>0$. The Weierstrass $\wp(z)$ and its derivative $\wp^{\prime}(z)$ satisfy an equation

$$
\wp^{\prime}(z)^{2}=4 \wp^{2}(x)-g_{4} \wp(z)-g_{6},
$$

where the precise formulas for the $g_{i}$ are not important for us. Thus $\sigma: z \mapsto$ $\left(\wp(z): \wp^{\prime}(z): 1\right)$ gives an isomorphism

$$
\sigma: \mathbb{C} / \Lambda \cong C_{\tau}:=\left(y^{2} w=4 x^{3}-g_{4} x w^{2}-g_{6} w^{3}\right) \subset \mathbb{P}_{x y w}^{2} .
$$

The origin is mapped to the point at infinity which is an inflection point of the curve.

For any $z_{0} \in \mathbb{C}$, we can also use $\sigma_{z_{0}}: z \mapsto\left(\wp\left(z-z_{0}\right): \wp^{\prime}\left(z-z_{0}\right): 1\right)$ to get another isomorphism

$$
\sigma_{z_{0}}: \mathbb{C} / \Lambda \cong C_{\tau}=\left(y^{2} w=4 x^{3}-g_{4} x w^{2}-g_{6} w^{3}\right) \subset \mathbb{P}_{x y w}^{2} .
$$

Here the point $z_{0} \in \mathbb{C}$ is mapped to the inflection point at infinity.

A linear automorphism of $\mathbb{P}_{x y w}^{2}$ preserves inflection points, so the automorphism $\sigma_{z_{0}} \circ \sigma^{-1}: C_{\tau} \cong C_{\tau}$ cannot be linear if $\sigma\left(z_{0}\right)$ is not an inflection point.

With a little more work, we get that $\sigma_{z_{0}} \sigma^{-1}: C_{\tau} \cong C_{\tau}$ is linear iff $z_{0} \in \frac{1}{3} \Lambda$.

Example 32. A more geometric way of obtaining a nonlinear automorphism of a smooth plane cubic $C \subset \mathbb{P}^{2}$ is the following. Pick a point $p_{0} \in C$ and for $p \in C$ let $\tau_{p_{0}}(p)$ denote the third intersection point of the line $\left\langle p, p_{0}\right\rangle$ with $C$. Thus $\tau_{p_{0}}$ is an involution of $C$, and it is linear iff $p_{0}$ is an inflection point of $C$.

In the description of Side Example 31, we can get $\tau_{p_{0}}$ as $z \mapsto 2 z_{0}-z$, where $\sigma\left(z_{0}\right)=p_{0}$.

The third exceptional case is $d=4$ and $n=2$, that is, degree 4 surfaces in $\mathbb{P}^{3}$. Here there are many known examples of nonlinear isomorphisms, but these are not easy to find and we do not have a complete description. A rather large set of examples is the following.

Example 33. Start with $\mathbb{P}_{\mathbf{x}}^{3} \times \mathbb{P}_{\mathbf{y}}^{3}$ and four bilinear hypersurfaces given by equations $H_{k}:=\left(\sum_{i j} a_{i j}^{k} x_{i} y_{j}=0\right)$. Set $S:=H_{1} \cap \cdots \cap H_{4}$. In order to compute its projection to $\mathbb{P}_{\mathbf{x}}^{3}$, we view the $H_{k}$ as linear equations in the $\mathbf{y}$-variables. The coefficient matrix is

$$
\mathbf{B}=\left(b_{k j}\right) \quad \text { where } \quad b_{k j}=\sum_{i} a_{i j}^{k} x_{i} .
$$

A given point $\mathbf{p} \in \mathbb{P}_{\mathbf{x}}^{3}$ is in $\pi_{\mathbf{x}}(S)$ iff $\mathbf{B}(\mathbf{p}) \mathbf{y}^{t}=0$ has a nonzero solution $\mathbf{y}$ and this in turn holds iff $\operatorname{det} \mathbf{B}(\mathbf{p})=0$. Thus

$$
\pi_{\mathbf{x}}(S)=(\operatorname{det} \mathbf{B}(\mathbf{x})=0) \subset \mathbb{P}_{\mathbf{x}}^{3} .
$$

Similarly, the image of the projection to $\mathbb{P}_{\mathbf{y}}^{3}$ is given by

$$
\pi_{\mathbf{y}}(S)=(\operatorname{det} \mathbf{C}(\mathbf{y})=0) \quad \text { where } \quad \mathbf{C}=\left(c_{k i}\right)=\left(\sum_{j} a_{i j}^{k} y_{j}\right) .
$$

It is not hard to check that, for general $a_{i j}^{k}$, the projections give isomorphisms

$$
S \cong(\operatorname{det} \mathbf{B}(\mathbf{x})=0) \text { and } S \cong(\operatorname{det} \mathbf{C}(\mathbf{y})=0) \text {. }
$$


In particular, the quartic surfaces $(\operatorname{det} \mathbf{B}=0)$ and $(\operatorname{det} \mathbf{C}=0)$ are isomorphic to each other. By Cramer's rule the isomorphism between them can be given by the coordinate functions

$$
\phi_{j}(\mathbf{x})=(-1)^{j-1} \operatorname{det} \mathbf{B}_{4 j}(\mathbf{x}),
$$

where $\mathbf{B}_{4 j}$ is the submatrix obtained by removing the fourth row and $j$ th column. So the $\phi_{j}(\mathbf{x})$ are cubic polynomials.

In principle it could happen that $(\operatorname{det} \mathbf{B}(\mathbf{x})=0)$ and $(\operatorname{det} \mathbf{C}(\mathbf{y})=0)$ are isomorphic by a linear isomorphism. This does not occur for general $a_{i j}^{k}$, though this needs proof. See [SS16, Ogu16 for this and other such examples.

One can play the same game with $\mathbb{P}_{\mathbf{x}}^{n+1} \times \mathbb{P}_{\mathbf{y}}^{n+1}$ and $n+2$ bilinear hypersurfaces. It turns out that for $n \geq 3$ the projections are birational but they are not isomorphisms, and the image hypersurfaces are always singular. Nonetheless, this construction gives very interesting examples of mildly singular (in fact with terminal singularities) hypersurfaces that are birational to each other in an unexpected way.

34. Comments on the Proof of Theorem 30, The theorem is an easy consequence of some big theorems, and one should prove the big theorems. I give only the bare outlines and references.

Assume first that $\operatorname{dim} X \geq 3$. The main point is to show that rational maps $X \rightarrow \mathbb{P}^{m}$ have a unique optimal representation, analogous to (9,5). We start with a single rational function.

Claim 34.1. Let $X \subset \mathbb{P}^{n+1}$ be a smooth hypersurface of dimension $n \geq 3$. Let $\phi$ be a rational function on $X$. Then there are homogeneous polynomials $p, q$ such that $\phi=\left.(p / q)\right|_{X}$ and

(a) the (closure of the) zero set of $\phi$ is exactly $X \cap(p=0)$ and

(b) the (closure of the) polar set of $\phi$ is exactly $X \cap(q=0)$.

A key observation of Lefschetz is that although Claim 34, 1 is algebraic, the hardest part of it is topological. The closure of the zero set of $\phi$ is a subset $Z \subset X(\mathbb{C})$ of real codimension 2 . It has a homology class in $H_{2 n-2}(X(\mathbb{C}), \mathbb{Z})$, we prefer to use instead its dual cohomology class $[Z] \in H^{2}(X(\mathbb{C}), \mathbb{Z})$. So the topological version of Claim $\left[34,1\right.$ asks whether $[Z] \in H^{2}(X(\mathbb{C}), \mathbb{Z})$ is obtained as the restriction of an element of $H^{2}\left(\mathbb{C P}^{n+1}, \mathbb{Z}\right)$. The following is a consequence of the Lefschetz hyperplane theorem; see Lef50, Gro68 or [GH78, p.156] for proofs.

Theorem 34,2. Let $X \subset \mathbb{P}^{n+1}$ be a smooth hypersurface. Then the natural map

$$
H^{i}\left(\mathbb{C P}^{n+1}, \mathbb{Z}\right) \rightarrow H^{i}(X(\mathbb{C}), \mathbb{Z})
$$

is an isomorphism for $i<n$.

We already hinted that Claim 34, 1 needs the case $i=2$ (hence the assumption $n \geq 3$ ), and going from the topological to the algebraic version also needs (34,2.a) for $i=1$.

From Claim 34, 1 it is easy to derive the following generalization of (9,5).

Claim 34.3. Let $X \subset \mathbb{P}^{n+1}$ be a smooth hypersurface of dimension $n \geq 3$, and let $\Phi: X \rightarrow \mathbb{P}^{m}$ be a nonconstant morphism. Then it can be written as

$$
\Phi=\left(P_{0}(\mathbf{x}): \cdots: P_{m}(\mathbf{x})\right), \quad \text { where }
$$

(a) the $P_{i}$ are homogeneous polynomials of the same degree $d$ and

(b) they do not have a common zero on $X$. 
Finally, it is straightforward to conclude that if $\Phi$ is an injection, then the degree of its image is $d^{\operatorname{dim} X} \cdot \operatorname{deg} X$. Thus $X \hookrightarrow \mathbb{P}^{n+1}$ is the unique smallest degree embedding of $X$ into any projective space, up to linear coordinate changes.

As we saw in Warning 11]2, Claim 34, 1 does not hold if $\operatorname{dim} X=2$, and, by Example 33, the case $\operatorname{dim} X=2, \operatorname{deg} X=4$ is exceptional. A different approach for all cases where $\operatorname{dim} X \geq 2, \operatorname{deg} X \neq \operatorname{dim} X+2$, will be discussed in paragraph 43 ,

Finally, for $n=1$, a theorem of Noether Noe1882, Sec.5] describes all low degree embeddings of a plane curve into some projective space. A particular case of this says that an irreducible plane curve of degree $\geq 4$ has a unique embedding into $\mathbb{P}^{2}$. A complete proof is given by Hartshorne [Har86, see also [ACGH85, p.56].

\section{NonRATionality OF LARGE DEGREE HyPERSURFACES}

We start with the following answer to Question 22.

Theorem 35. Let $X \subset \mathbb{P}^{n+1}$ be a smooth hypersurface of degree $d$. If $d \geq n+2$, then $X$ is not rational. More strongly, the image of every rational map $\Phi: \mathbb{P}^{n} \rightarrow X$ has dimension $\leq n-1$.

The stronger property is usually stated as " $X$ is not unirational". The theorem will be a direct consequence of Propositions 40 and 42, and the same method also answers Question 23 for large degree hypersurfaces.

Theorem 36. Let $X_{1}, X_{2} \subset \mathbb{P}^{n+1}$ be smooth hypersurfaces of degrees $d_{1}, d_{2}$ that are birational to each other. Assume that $d_{1} \geq n+3$. Then $d_{1}=d_{2}$ and the $X_{i}$ can be obtained from each other by a linear change of coordinates.

In order to prove Theorem 35, we need to study algebraic differential forms.

Definition 37. Let $X$ be a smooth hypersurface of dimension $n$. As we noted in Definition 6, it is also a real manifold of dimension 2n. An algebraic differential $m$-form is a differential $m$-form that locally can be written as linear combination of terms

(37, 1$)$

$$
\phi(\mathbf{x}) \cdot d \psi_{1}(\mathbf{x}) \wedge \cdots \wedge d \psi_{m}(\mathbf{x})
$$

where $\phi$ and the $\psi_{i}$ are rational functions. An algebraic differential form is defined at a point $p \in X$ if it can be written with summands as in 371) where $\phi$ and the $\psi_{i}$ are all defined at $p$.

We will be especially interested in $n$-forms, which are the algebraic volume forms. (It is not hard to check that if $\sigma$ is an algebraic volume form, then $\sigma \wedge \bar{\sigma}$ is a usual volume form, (up to a power of $\sqrt{-1}$ ), albeit possibly with zeros and poles.)

Example 38 (Algebraic volume forms on $\mathbb{C}^{n}$ ). On $\mathbb{C}^{n}$ the standard volume form is $d z_{1} \wedge \cdots \wedge d z_{n}$. If $\psi$ is a rational function, then

$$
d \psi=\sum_{i} \frac{\partial \psi}{\partial z_{i}} d z_{i}
$$

hence every algebraic volume form can be written as

$$
\sigma:=\phi(\mathbf{z}) \cdot d z_{1} \wedge \cdots \wedge d z_{n},
$$

where $\phi$ is a rational function. This in undefined precisely where $\phi$ is undefined. Thus, as we noted in Definition 8, either $\sigma$ is everywhere defined or it is undefined exactly along a hypersurface. 
The projective case behaves quite differently.

Lemma 39. There are no everywhere defined algebraic volume forms on $\mathbb{P}^{n}$.

Proof. We use projective coordinates $X_{0}: \cdots: X_{n}$ and show the claim using just two of the affine charts. Let these be

$$
\begin{aligned}
& \left(x_{1}, \ldots, x_{n}\right)=\left(X_{1} / X_{0}, \ldots, X_{n} / X_{0}\right), \\
& \left(y_{0}, \ldots, y_{n-1}\right)=\left(X_{0} / X_{n}, \ldots, X_{n-1} / X_{n}\right) .
\end{aligned}
$$

Thus, we can transition between these charts by the formulas

$$
x_{1}=\frac{y_{1}}{y_{0}}, \ldots, x_{n-1}=\frac{y_{n-1}}{y_{0}}, x_{n}=\frac{1}{y_{0}} .
$$

This gives that

$$
d x_{1} \wedge \cdots \wedge d x_{n}=d \frac{y_{1}}{y_{0}} \wedge \cdots \wedge d \frac{y_{n-1}}{y_{0}} \wedge d \frac{1}{y_{0}}=\frac{(-1)^{n}}{y_{0}^{n+1}} \cdot d y_{0} \wedge \cdots \wedge d y_{n-1} .
$$

By Example 38, any algebraic volume form on the first chart $\mathbb{C}_{\mathbf{x}}^{n}$ is of the form $f(\mathbf{x}) \cdot d x_{1} \wedge \cdots \wedge d x_{n}$. On the second chart $\mathbb{C}_{\mathbf{y}}^{n}$ it becomes

$$
\frac{(-1)^{n}}{y_{0}^{n+1}} f\left(\frac{y_{1}}{y_{0}}, \ldots, \frac{y_{n-1}}{y_{0}}, \frac{1}{y_{0}}\right) \cdot d y_{0} \wedge \cdots \wedge d y_{n-1} .
$$

Thus, it has a pole of order $\geq n+1$ along $\left(y_{0}=0\right)$, so it is not defined there.

Proposition 40. Let $X$ be a smooth projective hypersurface that has an everywhere defined, nonzero, algebraic volume form $\sigma_{X}$. Then $X$ is not rational. In fact, there is not even a rational map $\Phi: \mathbb{P}^{n} \rightarrow X$ with dense image.

Proof. By Claim 9,6 (or the more general Theorem 12), there is a closed subset $Z \subset \mathbb{P}^{n}$ of codimension $\geq 2$ such that $\Phi$ is defined on $\mathbb{P}^{n} \backslash Z$. Thus $\Phi^{*} \sigma_{X}$ is an algebraic volume form on $\mathbb{P}^{n} \backslash Z$. By the purity of poles noted in Example 38, $\Phi^{*} \sigma$ must extend to an everywhere defined algebraic volume form on $\mathbb{P}^{n}$. This is impossible by Lemma 39 .

Side Remark. The second part of Proposition 40 is one result that is quite different in positive characteristic. If we are in characteristic $p$, then $\left(x^{p}\right)^{\prime}=p x^{p-1}$ is identically 0 . Thus the pullback of a nonzero algebraic volume form can be identically zero.

Thus, should we write down algebraic volume forms on hypersurfaces. We start with the affine case.

41. Algebraic volume forms on affine hypersurfaces. Let $X \subset \mathbb{C}^{n+1}$ be a hypersurface. As in Sha74, Sec.III.6.4], it is quite easy to write down a nowhere zero volume form on $X^{\mathrm{sm}}$, the smooth part of $X$.

Claim 41,1. For any hypersurface $X=(h=0) \subset \mathbb{C}^{n+1}$, the volume forms

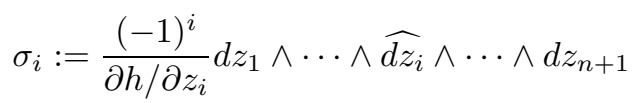

patch together to a volume form $\sigma_{X}$ that is defined and nowhere zero on $X^{\mathrm{sm}}$. 
Proof. By definition, $\left.h\right|_{X}=0$, thus $d h=\sum_{j} \frac{\partial h}{\partial z_{i}} d z_{j}$ is also zero on $X$. Wedging it with all the $d z_{i}$ except $d z_{i_{1}}$ and $d z_{i_{2}}$ gives that $\sigma_{i_{1}}=\sigma_{i_{2}}$. Thus, the $\sigma_{i}$ patch together to a rational volume form on $X$.

It is clear that $\sigma_{i}$ is defined and nowhere zero on the open set where $\partial h / \partial z_{i} \neq 0$. Thus at least one of the $\sigma_{i}$ is defined at a smooth point of $X$, so $\sigma_{X}$ is defined and nowhere zero on $X^{\mathrm{sm}}$.

As a counterpart/consequence of Claim [10,2 we obtain the following.

Claim 41.3. Let $X \subset \mathbb{A}^{n+1}$ be a smooth hypersurface and $\rho$ an everywhere defined algebraic volume form on $X$. Then it can be written as $\rho=p(\mathbf{x}) \sigma_{X}$, where $p$ is a polynomial on $\mathbb{A}^{n+1}$.

We already noted the following analogue of Claim 10, for $X=\mathbb{C}^{n}$, the general case is proved similarly using Claim [10,1.

Purity Principle 41,4. Let $\rho$ be an algebraic volume form on a smooth hypersurface $X$. Then either $\rho$ is everywhere defined or it is not defined along a codimension 1 subset of $X$.

Proposition 42. Let $X \subset \mathbb{P}^{n+1}$ be a smooth hypersurface of degree $d$. If $d \geq n+2$, then it has a nonzero, everywhere defined, algebraic volume form.

More precisely, the vector space of everywhere defined, algebraic volume forms on $X$ is naturally isomorphic to the vector space of homogeneous polynomials of degree $d-n-2$ in $n+2$ variables.

Proof. We can combine the computations of paragraph 41 and Lemma 39 to determine the volume forms on smooth hypersurfaces, but the following way may be quicker.

As we discussed in Definition [5, $\mathbb{C P}^{n+1}$ is the quotient of $\mathbb{C}^{n+2} \backslash\{0\}$ by the $\mathbb{C}^{*}$-action

$$
m_{\lambda}:\left(X_{0}, \ldots, X_{n+1}\right) \mapsto\left(\lambda X_{0}, \ldots, \lambda X_{n+1}\right),
$$

which is induced by the vector field $v_{X}:=\sum X_{i} \frac{\partial}{\partial X_{i}}$.

We can thus think of a volume form on $\mathbb{C P}^{n+1}$ as a $\mathbb{C}^{*}$-equivariant volume form on $\mathbb{C}^{n+2} \backslash\{0\}$, contracted by the vector field $v_{X}$. By Claim 41, 3 every volume form on $\mathbb{C}^{n+2} \backslash\{0\}$ extends to a volume form on $\mathbb{C}^{n+2}$.

Next, let $X=X(H) \subset \mathbb{C P}^{n+1}$ be a smooth hypersurface of degree $d$, and let $C_{X}:=(H=0) \subset \mathbb{C}^{n+2}$ be the cone $(5,5)$ over it. Denote the cone minus its vertex by $C_{X}^{\circ}$. We can thus view a volume form on $X$ as a $\mathbb{C}^{*}$-equivariant volume form on $C_{X}^{\circ}$ contracted by $v_{X}$. We wrote down a nowhere zero volume form on $C_{X}^{\circ}$ in [41,2). Choose the chart where it is given by

$$
\Sigma_{X}:=\left\{\frac{ \pm 1}{\partial H / \partial X_{n+1}} d X_{0} \wedge \cdots \wedge d X_{n}\right\}
$$

In particular,

$$
m_{\lambda}^{*} \Sigma_{X}=\lambda^{n+1-(d-1)} \Sigma_{X} .
$$

Thus if $G$ is any homogeneous polynomial, then

$$
m_{\lambda}^{*}\left(G \Sigma_{X}\right)=\lambda^{\operatorname{deg} G+n+2-d} \cdot G \Sigma_{X} .
$$

Hence $G \Sigma_{X}$ is $\mathbb{C}^{*}$-invariant iff $\operatorname{deg} G=d-n-2$. 
Let us work out explicitly what we get in terms of the forms $\sigma_{i}$ given in (41)2). Contracting $G \Sigma_{X}$ by $v_{X}$ gives

$$
\sum X_{i} \frac{\partial}{\partial X_{i}}\left(G \Sigma_{X}\right)=\sum_{i=0}^{n} X_{i} G \cdot \frac{d X_{0} \wedge \cdots \wedge \widehat{d X}_{i} \wedge \cdots \wedge d X_{n}}{\partial H / \partial X_{n+1}} .
$$

We need to pull this back to $X$ by the map $\left(x_{1}, \ldots, x_{n+1}\right) \mapsto\left(1: x_{1}: \cdots: x_{n+1}\right)$. The pullback of $d X_{0}$ is then 0 , and the only term that survives is

$$
G\left(1, x_{1}, \ldots, x_{n+1}\right) \frac{d x_{1} \wedge \cdots \wedge d x_{n}}{\partial H\left(1, x_{1}, \ldots, x_{n+1}\right) / \partial x_{n+1}}=G\left(1, x_{1}, \ldots, x_{n+1}\right) \cdot \sigma_{n+1},
$$

where $\sigma_{n+1}$ is as in (41,2).

43. Comments on the Proofs of Theorems 30 and 36. We proved in Proposition 42 that the vector space of homogeneous polynomials of degree $\operatorname{deg} X-n-2$ in $n+2$ variables (an invariant of the embedding $X \hookrightarrow \mathbb{P}^{n+1}$ ) is naturally isomorphic to the vector space of everywhere defined algebraic volume forms on $X$ (an intrinsic invariant of $X$ ).

We are completely done with both theorems if $d=n+3$. Then the volume forms tell us the linear polynomials, and they define the embedding $X \hookrightarrow \mathbb{P}^{n+1}$.

We are almost done if $d>n+3$; the missing ingredient is that $H^{2}(X(\mathbb{C}), \mathbb{Z})$ is torsion free for $\operatorname{dim} X \geq 2$. This is another special case of the Lefschetz hyperplane theorem.

If $d<n+3$, then we can use the duals of holomorphic volume forms. These are wedge products of tangent vector fields. We can pull back a vector field by an isomorphism but not by an arbitrary map. This is why Theorem 30 works for $d<n+3$ but Theorem 36 does not.

\section{NonRationality of LOW DEgReE HyPERSuRfaceS}

In the previous section we proved that a smooth hypersurface $X \subset \mathbb{P}^{n+1}$ of degree $\geq n+2$ is not rational. Here we discuss what is known when the degree is $\leq n+1$. Most of the results use different - and much more advanced-methods, so we give the statements only, with barely a hint of how they can be proved.

We proved in Theorem 7 that every irreducible quadric hypersurface is rational over $\mathbb{C}$ and, by Corollary 25, every smooth cubic surface is rational.

We already noted that smooth cubic 3 -folds are all nonrational, and the same holds for smooth quartic 3-folds IM71; we say more about this in Section 9 .

Another method, using differential forms over fields of positive characteristic, was introduced in Kol95. We proved in [42,5) that if $\operatorname{deg} X<n+2$, then there are no everywhere defined $n$-forms on $X$. One can show that there are also no everywhere defined algebraic $r$-forms on $X$ for $r \leq n$.

It turns out that while the first of these claims continues to hold over fields of positive characteristic, there are some singular hypersurfaces that carry $(n-1)$ forms. After dealing with some technical issues involving these singularities, one can sometimes conclude that these hypersurfaces are nonrational. However, this happens in positive characteristic. Nonetheless, one can use some general theorems going back to Matsusaka and Mumford MM64 to get similar conclusions over $\mathbb{C}$.

A serious drawback is that we can prove nonrationality only for hypersurfaces $X=\left(\sum_{I} a_{I} x^{I}=0\right)$ whose coefficients satisfy countably many conditions of the 
form $p_{j}\left(a_{I}\right) \neq 0$, where the $p_{j}$ are (pretty much unknown) polynomials. We refer to such hypersurfaces as very general.

Theorem 44 ([Kol95]). Very general hypersurfaces $X_{d} \subset \mathbb{P}^{n+1}$ of degree $d \geq \frac{2}{3} n+3$ are not rational. Moreover, $X_{d}$ is not birational to any product $\mathbb{P}^{1} \times Y$, where $Y$ is a hypersurface (or variety) of dimension $n-1$.

I stress that we really do not know which hypersurfaces are covered by the theorem, though some concrete examples were written down in Kol96, Sec.V.5].

Another argument, this time using degeneration to certain singular varieties and the topology of their resolution, was introduced by Voisin Voi15. It was used by many authors to prove nonrationality of varieties. For hypersurfaces the strongest result is proved by $\mathrm{Sch18}$.

Theorem 45 (Schreieder). Very general hypersurfaces $X_{d} \subset \mathbb{P}^{n+1}$ of degree $d \geq$ $2+\log _{2} n$ are not rational for $n \geq 3$.

\section{Rigidity OF LOW DEGREE HYPERSURFACES}

Much less is known about Question 20 for hypersurfaces $X_{d} \subset \mathbb{P}^{n+1}$ whose degree is $3 \leq d \leq n+1$. We studied the degree 3 case in Section 5 . The answer in the $d=n+1$ case is one of the crowning achievements of the Noether-Fano rigidity theory.

I call it the Noether-Fano-Segre-Iskovskikh-Manin-Pukhlikov-Corti-CheltsovdeFernex-Ein-Mustaţă-Zhuang theorem, although the contributions of Fano, Iskovskikh, and Pukhlikov were the most substantial. See Kol18 for a detailed survey.

Theorem 46. Let $X \subset \mathbb{P}^{n+1}$ be a smooth hypersurface of degree $n+1$. Assume that $n \geq 3$. Then $X$ is not birational to any other smooth hypersurface.

47. A short history of Theorem 46. The first similar result is Max Noether's description of all birational maps $\mathbb{P}^{2} \rightarrow \mathbb{P}^{2}$ [Noe1870, whose method formed the basis of all further developments.

Theorem 46 was first stated by Fano for 3-folds Fan1908, Fan1915. His arguments contain many of the key ideas, but they also have gaps. I call this approach the Noether-Fano method. The first complete proof for 3-folds, along the lines indicated by Fano, is in Iskovskikh and Manin IM71. Iskovskikh and his school used this method to prove similar results for many other 3-folds; see Isk79, Sar80,IP99, Isk01]. This approach was gradually extended to higher dimensions by Pukhlikov Puk87,Puk98, Puk02 and Cheltsov Che00. These results were complete up to dimension 8 , but needed some additional general position assumptions in higher dimensions. A detailed survey of this direction is in Puk13.

The Noether-Fano method and the Minimal Model Program were brought together by Corti [Cor95]. (See [Kol87] for a by-now outdated but elementary introduction and [KM98] for a detailed treatment.) Corti's technique has been very successful in many cases, especially for 3-folds; see [CR00] for a detailed study and [KSC04, Chap.5] for an introduction. However, usually one needs some special tricks to make the last steps work, and a good higher dimensional version proved elusive for a long time.

New methods involving multiplier ideals were introduced by de Fernex, Ein, and Mustaţă dFEM03; these led to a more streamlined proof that worked up to dimension 12 . The proof of Theorem 46 was finally completed by de Fernex dF16]. 
The recent paper of Zhuang [Zhu18] makes the final step of the Corti approach much easier in higher dimensions. The papers [SZ18, Zhu18, LZ18] contain more general results and applications.

\section{Connections With the Classification of varieties}

So far I have studiously avoided assuming prior knowledge of algebraic geometry. However, in order to explain the place of these results in algebraic geometry, it becomes necessary to use the basic theory, as in [Sha74, Vol.I]. In particular we need to know the notions of ampleness and canonical class $K_{X}$.

The classification theory of algebraic varieties - developed by Enriques for surfaces and extended by Iitaka and then Mori to higher dimensions - says that every variety can be built from three basic types:

- General type: $K_{X}$ is ample,

- Calabi-Yau: $K_{X}$ is trivial, and

- Fano: $-K_{X}$ is ample;

see Kol14 for an introduction. Moreover, in the Fano case the truly basic ones are those that satisfy $H^{2}(X(\mathbb{C}), \mathbb{Z}) \cong \mathbb{Z}$. (It is better think of the last condition as $X$ having class number equal to 1 . That is, every divisor $D$ on $X$ is linearly equivalent to a (possibly rational) multiple of $-K_{X}$.)

The computations of Proposition 42 show that if $X \subset \mathbb{P}^{n+1}$ is a smooth hypersurface, then it is of general type iff $\operatorname{deg} X \geq n+3$, it is Calabi-Yau iff $\operatorname{deg} X=n+2$, and it is Fano iff $\operatorname{deg} X \leq n+1$.

As in Theorem 36, one can see that if two varieties $X_{1}, X_{2}$ on the basic type list are birationally equivalent, then they have the same type. Furthermore, in the general type case they are even isomorphic; this generalizes Theorem 36. In the Calabi-Yau case, $X_{1}$ and $X_{2}$ need not be isomorphic, but the possible birational maps between two Calabi-Yau varieties are reasonably well understood, especially in dimension 3; see, for example, Kol89, Kol91, CK16.

It is very useful to think of Fano varieties as representatives of rationally connected varieties, but this would have taken us in another direction; see [Kol01] for an overview and Kol96, AK03 for more detailed treatments.

Thus, from the general point of view, for low degree cases, the best variant of Question 20 is the following.

Definition 48. A Fano variety $X$ with class number 1 is called weakly superrigid if every birational map $\Phi: X \rightarrow Y$ to another Fano variety $Y$ with class number 1 is an isomorphism.

Question 49. Which Fano varieties are weakly superrigid?

The adjective "weakly" is not standard; I use it just to avoid further definitions. The correct definition of superrigid allows $Y$ to have terminal singularities and to be a Mori fiber space; see Puk95. Che05]. The proofs in the Noether-Fano theory are designed to prove superrigidity. However, there should be many varieties that are weakly superrigid but not superrigid; see Question 54.

\section{Open problems about hypersurfaces}

The following questions are stated in the strongest forms that are consistent with known examples. I have no reason to believe that the answer to any of them 
is positive, and there may well be rather simple counterexamples. As far as I know, there has been very little work on low degree hypersurfaces beyond cubics in dimension 4.

All the known rationality constructions work for even dimensional cubics only, so the following is still open.

Question 50. Is there any odd dimensional, smooth, rational cubic hypersurface?

We leave to the reader the easy task of generalizing Proposition 24 to the following higher dimensional version.

Proposition 51. Let $X \subset \mathbb{P}^{2 n+1}$ be a cubic hypersurface that contains two disjoint linear subspaces of dimension $n$. Then $X$ is rational.

This leaves the following open.

Question 52. Is the general even dimensional, smooth, cubic hypersurface of dimension $\geq 4$ nonrational?

We saw that quadrics are rational and Proposition 51 gives examples of cubic hypersurfaces that are rational. However, no rationality construction is known for smooth hypersurfaces of higher degree. Example (21]2) gives higher degree rational hypersurfaces $X_{2 d+1}^{2 n} \subset \mathbb{P}^{2 n+1}$, but they are always singular. However, the singularities are mild when $d \leq n$. To be precise, they are canonical if $d \leq n$ and terminal if $d \leq n-1$. (See [KM98, Sec.2.3] or [Kol13] for introductions to such singularities.) Thus, the following problem is still open.

Question 53. Is there any smooth, rational hypersurface of degree $\geq 4$ ?

Understanding birational maps between Fano hypersurfaces is even harder. Theorem 46 deals with smooth hypersurfaces $X \subset \mathbb{P}^{n+1}$ of degree $n+1$.

Question 54. Is every birational map between smooth hypersurfaces of degree $\geq 5$ an isomorphism?

Here $\geq 5$ is necessary since there are some smooth quartics with nontrivial birational maps.

Example 55. Let $X \subset \mathbb{P}^{2 n+1}$ be a quartic hypersurface that contains two disjoint linear subspaces $L_{1}, L_{2}$ of dimension $n$.

As in Proposition 24. for every $\mathbf{p} \in \mathbb{P}^{2 n+1} \backslash\left(L_{1} \cup L_{2}\right)$ there is a unique line $\ell_{\mathbf{p}}$ through $\mathbf{p}$ that meets both $L_{1}, L_{2}$. This line meets $X$ in four points; two of these are on $L_{1}, L_{2}$. If $\mathbf{p} \in X$, then this leaves a unique fourth intersection point, call it $\Phi(\mathbf{p})$. Clearly, $\Phi$ is an involution which is not defined at $\mathbf{p}$ if either $\mathbf{p} \in L_{1} \cup L_{2}$ or if $\ell_{\mathbf{p}} \subset X$.

To get a concrete example, for $n \geq 2$ consider the smooth, quartic hypersurface

$$
X:=\left(x_{0}^{4}+\cdots+x_{2 n+1}^{4}=0\right) \subset \mathbb{P}^{2 n+1} .
$$

Then $\operatorname{Aut}(X)$ is finite (probably of order $4^{2 n+1} \cdot(2 n+2)$ !) but combining Example 26 with the above observation gives many birational involutions on $X$. Most likely these involutions generate an infinite subgroup of $\operatorname{Bir}(X)$. 


\section{ACKNOWLEDGMENTS}

These notes are based on lectures given at Northwestern University in April and May of 2018. The hospitality of Northwestern University, and especially of M. Popa, gave an ideal time to write them up in expanded form. I received many helpful comments and references from V. Cheltsov, A. Corti, T. de Fernex, L. Ein, Y. Liu, and B. Totaro.

\section{ABOut THE AUTHOR}

János Kollár got his PhD in 1984 with Teruhisa Matsusaka. After three years at Harvard as a junior fellow and twelve years at the University of Utah, he is now the Donner Professor of Science at Princeton University. His main research interest is algebraic geometry, especially the birational classification of higher dimensional algebraic varieties, but he also likes to explore applications of these ideas in combinatorics, commutative algebra, differential geometry, logic, and number theory. He is also very attached to his accent marks.

\section{REFERENCES}

[Abh77] S. S. Abhyankar, Lectures on expansion techniques in algebraic geometry, Tata Institute of Fundamental Research Lectures on Mathematics and Physics, vol. 57, Tata Institute of Fundamental Research, Bombay, 1977. Notes by Balwant Singh. MR.542446

[ACGH85] E. Arbarello, M. Cornalba, P. A. Griffiths, and J. Harris, Geometry of algebraic curves. Vol. I, Grundlehren der Mathematischen Wissenschaften [Fundamental Principles of Mathematical Sciences], vol. 267, Springer-Verlag, New York, 1985. MR770932

[AK03] C. Araujo and J. Kollár, Rational curves on varieties, Higher dimensional varieties and rational points (Budapest, 2001), Bolyai Soc. Math. Stud., vol. 12, Springer, Berlin, 2003, pp. 13-68, DOI 10.1007/978-3-662-05123-8_3. MR.2011743

[AL92] E. Andersén and L. Lempert, On the group of holomorphic automorphisms of $\mathbf{C}^{n}$, Invent. Math. 110 (1992), no. 2, 371-388, DOI 10.1007/BF01231337. MR.1185588

[AM75] S. S. Abhyankar and T. T. Moh, Embeddings of the line in the plane, J. Reine Angew. Math. 276 (1975), 148-166. MR0379502

[BHKV16] A. Beauville, B. Hassett, A. Kuznetsov, and A. Verra, Rationality problems in algebraic geometry, Lecture Notes in Mathematics, vol. 2172, Springer, Cham; Fondazione C.I.M.E., Florence, 2016. Lecture notes from the CIME-CIRM course held in Levico Terme, June 22-27, 2015; Edited by Rita Pardini and Gian Pietro Pirola; Fondazione CIME/CIME Foundation Subseries. MR 3587799

[CD94] A. D. R. Choudary and A. Dimca, Complex hypersurfaces diffeomorphic to affine spaces, Kodai Math. J. 17 (1994), no. 2, 171-178, DOI 10.2996/kmj/1138039958. MR.1282208

[CDP17] I. Cheltsov, A. Dubouloz, and J. Park, Super-rigid affine Fano varieties, Compos. Math. 154 (2018), no. 11, 2462-2484, DOI 10.1112/s0010437x18007534. MR3866510

[CG72] C. H. Clemens and P. A. Griffiths, The intermediate Jacobian of the cubic threefold, Ann. of Math. (2) 95 (1972), 281-356, DOI 10.2307/1970801. MR0302652

[Che00] I. A. Chel'tsov, On a smooth four-dimensional quintic (Russian, with Russian summary), Mat. Sb. 191 (2000), no. 9, 139-160, DOI 10.1070 /SM2000v191n09ABEH000511; English transl., Sb. Math. 191 (2000), no. 9-10, 1399-1419. MR 1805602

[Che05] I. A. Chel'tsov, Birationally rigid Fano varieties (Russian, with Russian summary), Uspekhi Mat. Nauk 60 (2005), no. 5(365), 71-160, DOI 10.1070 /RM2005v060n05ABEH003736; English transl., Russian Math. Surveys 60 (2005), no. 5, 875-965. MR.2195677

[CK16] A. Corti and A.-S. Kaloghiros, The Sarkisov program for Mori fibred Calabi-Yau pairs, Algebr. Geom. 3 (2016), no. 3, 370-384, DOI 10.14231/AG-2016-016. MR3504536

[Cle1866] A. Clebsch, Die Geometrie auf den Flächen dritter Ordnung (German), J. Reine Angew. Math. 65 (1866), 359-380, DOI 10.1515/crll.1866.65.359. MR.1579329 
[CLO92] D. Cox, J. Little, and D. O'Shea, Ideals, varieties, and algorithms: An introduction to computational algebraic geometry and commutative algebra, Undergraduate Texts in Mathematics, Springer-Verlag, New York, 1992. MR.1189133

[Cor95] A. Corti, Factoring birational maps of threefolds after Sarkisov, J. Algebraic Geom. 4 (1995), no. 2, 223-254. MR.1311348

[CR00] A. Corti and M. Reid (eds.), Explicit birational geometry of 3-folds, London Mathematical Society Lecture Note Series, vol. 281, Cambridge University Press, Cambridge, 2000. MR1798978

[dF16] T. de Fernex, Erratum to: Birationally rigid hypersurfaces [MR3049929], Invent. Math. 203 (2016), no. 2, 675-680, DOI 10.1007/s00222-015-0618-4. MR3455160

[dFEM03] T. de Fernex, L. Ein, and M. Mustaţă, Bounds for log canonical thresholds with applications to birational rigidity, Math. Res. Lett. 10 (2003), no. 2-3, 219-236, DOI 10.4310/MRL.2003.v10.n2.a9. MR1981899

[Dol12] I. V. Dolgachev, Classical algebraic geometry, Cambridge University Press, Cambridge, 2012. A modern view. MR2964027

[EH16] David Eisenbud and Joe Harris, 3264 and all that: A second course in algebraic geometry, Cambridge University Press, Cambridge, 2016. MR 3617981.

[Fan1908] Gino Fano, Sopra alcune varieta algebriche a tre dimensioni aventi tutti i generi nulli, Atti. Ac. Torino 43 (1908), 973-977.

[Fan1915] Gino Fano, Osservazioni sopra alcune varieta non razionali aventi tutti i generi nulli, Atti. Ac. Torino 50 (1915), 1067-1072.

[Fan43] G. Fano, Sulle forme cubiche dello spazio a cinque dimensioni contenenti rigate razionali del $4^{\circ}$ ordine (Italian), Comment. Math. Helv. 15 (1943), 71-80, DOI 10.1007/BF02565634. MR.0010433

[GH78] P. Griffiths and J. Harris, Principles of algebraic geometry, Wiley-Interscience [John Wiley \& Sons], New York, 1978. Pure and Applied Mathematics. MR507725

[Gro68] A. Grothendieck, Cohomologie locale des faisceaux cohérents et théorèmes de Lefschetz locaux et globaux (SGA 2) (French), North-Holland Publishing Co., Amsterdam; Masson \& Cie, Éditeur, Paris, 1968. Augmenté d'un exposé par Michèle Raynaud; Séminaire de Géométrie Algébrique du Bois-Marie, 1962; Advanced Studies in Pure Mathematics, Vol. 2. MR0476737

[Hal1695] Edmond Halley, An easie demonstration of the analogy of the logarithmick tangents to the meridian line or sum of the secants: With various methods for computing the same to the utmost exactness, Phil. Trans. Royal Soc. 19 (1695), 202-214.

[Har86] R. Hartshorne, Generalized divisors on Gorenstein curves and a theorem of Noether, J. Math. Kyoto Univ. 26 (1986), no. 3, 375-386, DOI 10.1215/kjm/1250520873. MR 857224

[Has99] B. Hassett, Some rational cubic fourfolds, J. Algebraic Geom. 8 (1999), no. 1, 103-114. MR.1658216

[IM71] V. A. Iskovskih and Ju. I. Manin, Three-dimensional quartics and counterexamples to the Lüroth problem (Russian), Mat. Sb. (N.S.) 86(128) (1971), 140-166. MR.0291172

[IP99] V. A. Iskovskikh and Yu. G. Prokhorov, Fano varieties, Algebraic geometry, V, Encyclopaedia Math. Sci., vol. 47, Springer, Berlin, 1999, pp. 1-247. MR 1668579

[Isk79] V. A. Iskovskih, Birational automorphisms of three-dimensional algebraic varieties (Russian), Current problems in mathematics, Vol. 12 (Russian), VINITI, Moscow, 1979, pp. 159-236, 239 (loose errata). MR.537686

[Isk01] V. A. Iskovskikh, Birational rigidity of Fano hypersurfaces in the framework of Mori theory (Russian, with Russian summary), Uspekhi Mat. Nauk 56 (2001), no. 2(338), 3-86, DOI 10.1070/RM2001v056n02ABEH000382; English transl., Russian Math. Surveys 56 (2001), no. 2, 207-291. MR.1859707

[Jel97] Z. Jelonek, A hypersurface which has the Abhyankar-Moh property, Math. Ann. 308 (1997), no. 1, 73-84, DOI 10.1007/s002080050065. MR.1446200

[KM98] J. Kollár and S. Mori, Birational geometry of algebraic varieties, Cambridge Tracts in Mathematics, vol. 134, Cambridge University Press, Cambridge, 1998. With the collaboration of C. H. Clemens and A. Corti; Translated from the 1998 Japanese original. MR 1658959

[Kol87] J. Kollár, The structure of algebraic threefolds: an introduction to Mori's program, Bull. Amer. Math. Soc. (N.S.) 17 (1987), no. 2, 211-273, DOI 10.1090/S0273-09791987-15548-0. MR903730 
[Kol89] J. Kollár, Flops, Nagoya Math. J. 113 (1989), 15-36, DOI 10.1017 /S0027763000001240. MR986434

[Kol91] J. Kollár, Flips, flops, minimal models, etc, Surveys in differential geometry (Cambridge, MA, 1990), Lehigh Univ., Bethlehem, PA, 1991, pp. 113-199. MR.1144527

[Kol95] J. Kollár, Nonrational hypersurfaces, J. Amer. Math. Soc. 8 (1995), no. 1, 241-249, DOI 10.2307/2152888. MR 1273416

[Kol96] J. Kollár, Rational curves on algebraic varieties, Ergebnisse der Mathematik und ihrer Grenzgebiete. 3. Folge. A Series of Modern Surveys in Mathematics [Results in Mathematics and Related Areas. 3rd Series. A Series of Modern Surveys in Mathematics], vol. 32, Springer-Verlag, Berlin, 1996. MR1440180

[Kol01] J. Kollár, Which are the simplest algebraic varieties?, Bull. Amer. Math. Soc. (N.S.) 38 (2001), no. 4, 409-433, DOI 10.1090/S0273-0979-01-00917-X. MR1848255

[Kol13] J. Kollár, Singularities of the minimal model program, Cambridge Tracts in Mathematics, vol. 200, Cambridge University Press, Cambridge, 2013. With a collaboration of Sándor Kovács. MR3057950

[Kol14] J. Kollár, The structure of algebraic varieties, Proceedings of ICM, Seoul, 2014, Vol. I., Kyung Moon SA, http://www.icm2014.org/en/vod/proceedings.html, 2014, pp. 395420 .

[Kol18] J. Kollár, The rigidity theorem of Noether-Fano-Segre-Iskovskikh-Manin-PukhlikovCorti-Cheltsov-de Fernex-Ein-Mustaţă-Zhuang, arXiv:1807.00863 (2018),

[KSC04] J. Kollár, K. E. Smith, and A. Corti, Rational and nearly rational varieties, Cambridge Studies in Advanced Mathematics, vol. 92, Cambridge University Press, Cambridge, 2004. MR2062787

[KT17] Maxim Kontsevich and Yuri Tschinkel, Specialization of birational types, arXiv:1708.05699 (2017)

[Kur16] S. Kuroda, Recent developments in polynomial automorphisms: the solution of $\mathrm{Na}$ gata's conjecture and afterwards [translation of MR3058805], Sugaku Expositions 29 (2016), no. 2, 177-201. MR3561400

[Lef50] S. Lefschetz, L'analysis situs et la géométrie algébrique (French), Gauthier-Villars, Paris, 1950. MR0033557

[LZ18] Yuchen Liu and Ziquan Zhuang, Birational superrigidity and K-stability of singular Fano complete intersections, arXiv e-prints (2018).

[Mac1916] F. S. Macaulay, The algebraic theory of modular systems, Cambridge Mathematical Library, Cambridge University Press, Cambridge, 1994. Revised reprint of the 1916 original; With an introduction by Paul Roberts. MR 1281612

[MM64] T. Matsusaka and D. Mumford, Two fundamental theorems on deformations of polarized varieties, Amer. J. Math. 86 (1964), 668-684, DOI 10.2307/2373030. MR0171778

[Mor40] U. Morin, Sulla razionalità dell'ipersuperficie cubica generale dello spazio lineare $S_{5}$ (Italian), Rend. Sem. Mat. Univ. Padova 11 (1940), 108-112. MR0020273

[Mum76] D. Mumford, Algebraic geometry. I: Complex projective varieties, Grundlehren der Mathematischen Wissenschaften, No. 221, Springer-Verlag, Berlin-New York, 1976. MR.0453732

[Nag72] M. Nagata, On automorphism group of $k[x, y]$, Kinokuniya Book-Store Co., Ltd., Tokyo, 1972. Department of Mathematics, Kyoto University, Lectures in Mathematics, No. 5. MR 0337962

[Noe1870] M. Noether, Ueber Flächen, welche Schaaren rationaler Curven besitzen (German), Math. Ann. 3 (1870), no. 2, 161-227, DOI 10.1007/BF01443982. MR1509694

[Noe1882] M. Noether, Zur Grundlegung der Theorie der algebraischen Raumcurven (German), J. Reine Angew. Math. 93 (1882), 271-318, DOI 10.1515/crll.1882.93.271. MR.1579926

[NS17] Johannes Nicaise and Evgeny Shinder, The motivic nearby fiber and degeneration of stable rationality, arXiv:1708.027901 (2017).

[Ogu16] K. Oguiso, Isomorphic quartic K3 surfaces in the view of Cremona and projective transformations, Taiwanese J. Math. 21 (2017), no. 3, 671-688, DOI 10.11650/tjm/7833. MR3661387

[Puk87] A. V. Pukhlikov, Birational isomorphisms of four-dimensional quintics, Invent. Math. 87 (1987), no. 2, 303-329, DOI 10.1007/BF01389417. MR870730

[Puk95] A. V. Pukhlikov, A remark on the theorem of V. A. Iskovskikh and Yu. I. Manin on a three-dimensional quartic, Trudy Mat. Inst. Steklov. 208 (1995), no. Teor. Chisel, 
Algebra i Algebr. Geom., 278-289, Dedicated to Academician Igor Rostislavovich Shafarevich on the occasion of his seventieth birthday (Russian). MR1730270

[Puk98] A. V. Pukhlikov, Birational automorphisms of Fano hypersurfaces, Invent. Math. 134 (1998), no. 2, 401-426, DOI 10.1007/s002220050269. MR1650332

[Puk02] A. V. Pukhlikov, Birationally rigid Fano hypersurfaces (Russian, with Russian summary), Izv. Ross. Akad. Nauk Ser. Mat. 66 (2002), no. 6, 159-186, DOI 10.1070/IM2002v066n06ABEH000413; English transl., Izv. Math. 66 (2002), no. 6, 1243-1269. MR.1970356

[Puk13] A. Pukhlikov, Birationally rigid varieties, Mathematical Surveys and Monographs, vol. 190, American Mathematical Society, Providence, RI, 2013. MR3060242

[Rei88] M. Reid, Undergraduate algebraic geometry, London Mathematical Society Student Texts, vol. 12, Cambridge University Press, Cambridge, 1988. MR.982494

[Rei97] M. Reid, Chapters on algebraic surfaces, Complex algebraic geometry (Park City, UT, 1993), IAS/Park City Math. Ser., vol. 3, Amer. Math. Soc., Providence, RI, 1997, pp. 3-159. MR.1442522

[RS17] F. Russo and G. Staglianò, Congruences of 5-secant conics and the rationality of some admissible cubic fourfolds, arXiv e-prints (2017).

[Sal1865] G. Salmon, A treatise on the analytic geometry of three dimensions. Vol. II, Fifth edition. Edited by Reginald A. P. Rogers, Chelsea Publishing Co., New York, 1965. MR0200123

[Sar80] V. G. Sarkisov, Birational automorphisms of conic bundles (Russian), Izv. Akad. Nauk SSSR Ser. Mat. 44 (1980), no. 4, 918-945, 974. MR587343

[Sch18] S. Schreieder, Stably irrational hypersurfaces of small slopes, arXiv e-prints (2018).

[Seg43] B. Segre, A note on arithmetical properties of cubic surfaces, J. London Math. Soc 18 (1943), 24-31, DOI 10.1112/jlms/s1-18.1.24. MR0009471

[Sha74] I. R. Shafarevich, Basic algebraic geometry, Springer-Verlag, New York-Heidelberg, 1974. Translated from the Russian by K. A. Hirsch; Die Grundlehren der mathematischen Wissenschaften, Band 213. MR0366917

[Sie69] C. L. Siegel, Topics in complex function theory. Vol. I: Elliptic functions and uniformization theory, Translated from the original German by A. Shenitzer and D. Solitar. Interscience Tracts in Pure and Applied Mathematics, No. 25, Wiley-Interscience A Division of John Wiley \& Sons, New York-London-Sydney, 1969. MR0257326

[Sil86] J. H. Silverman, The arithmetic of elliptic curves, Graduate Texts in Mathematics, vol. 106, Springer-Verlag, New York, 1986. MR817210

[SS03] E. M. Stein and R. Shakarchi, Complex analysis, Princeton Lectures in Analysis, vol. 2, Princeton University Press, Princeton, NJ, 2003. MR 1976398

[SS16] I. Shimada and T. Shioda, On a smooth quartic surface containing 56 lines which is isomorphic as a K3 surface to the Fermat quartic, Manuscripta Math. 153 (2017), no. 1-2, 279-297, DOI 10.1007/s00229-016-0886-3. MR3635983

[SU04] I. P. Shestakov and U. U. Umirbaev, The tame and the wild automorphisms of polynomial rings in three variables, J. Amer. Math. Soc. 17 (2004), no. 1, 197-227, DOI 10.1090/S0894-0347-03-00440-5. MR2015334

[Suz74] M. Suzuki, Propriétés topologiques des polynômes de deux variables complexes, et automorphismes algébriques de l'espace $\mathbf{C}^{2}$ (French), J. Math. Soc. Japan 26 (1974), 241-257, DOI 10.2969/jmsj/02620241. MR0338423

[SZ18] Charlie Stibitz and Ziquan Zhuang, K-stability of birationally superrigid Fano varieties, ArXiv e-prints (2018).

[vdE00] A. van den Essen, Polynomial automorphisms and the Jacobian conjecture, Progress in Mathematics, vol. 190, Birkhäuser Verlag, Basel, 2000. MR1790619

[Voi15] C. Voisin, Unirational threefolds with no universal codimension 2 cycle, Invent. Math. 201 (2015), no. 1, 207-237, DOI 10.1007/s00222-014-0551-y. MR.3359052

[Zhu18] Ziquan Zhuang, Birational superrigidity and K-stability of Fano complete intersections of index one (with an appendix written jointly with Charlie Stibitz), arXiv e-prints (2018).

Department of Mathematics, Princeton University, Princeton, New Jersey 085441000

Email address: kollar@math.princeton.edu 